\title{
Expression profile analysis identifies IER3 to predict overall survival and promote lymph node metastasis in tongue cancer
}

\author{
Fang Xiao ${ }^{1}$, Yinhua Dai ${ }^{1}$, Yujiao Hu${ }^{1}$, Mengmeng $\mathrm{Lu}^{2^{*}}$ (10 and Qun Dai ${ }^{1 *}$
}

\begin{abstract}
Background: Lymph node metastasis is one of the most important factors affecting the prognosis of tongue cancer, and the molecular mechanism regulating lymph node metastasis of tongue cancer is poorly known.

Methods: The gene expression dataset GSE2280 and The Cancer Genome Atlas (TCGA) tongue cancer dataset were downloaded. R software was used to identify the differentially expressed hallmark gene sets and individual genes between metastatic lymph node tissues and primary tongue cancer tissues, and the Kaplan-Meier method was used to evaluate the association with overall survival. The screening and validation of functional genes was performed using western blot, q-PCR, CCK-8, migration and invasion assays, and lymphangiogenesis was examined by using a tube formation assay.

Results: Thirteen common hallmark gene sets were found based on Gene Set Variation Analysis (GSVA) and then subjected to differential gene expression analysis, by which 76 deregulated genes were found. Gene coexpression network analysis and survival analysis further confirmed that IER3 was the key gene associated with the prognosis and lymph node metastasis of tongue cancer patients. Knockdown of IER3 with siRNA inhibited the proliferation, colony formation, migration and invasion of Tca-8113 cells in vitro and it also inhibited the secretion and expression of VEGF-C in these cells. The culture supernatant of Tca-8113 cells could promote lymphangiogenesis and migration of lymphatic endothelial cells, and knockdown of IER3 in Tca-8113 cells suppressed these processes.
\end{abstract}

Conclusion: Our study demonstrated that IER3 plays important roles in lymphangiogenesis regulation and prognosis in tongue cancer and might be a potential therapeutic target.

Keywords: Gene set variation analysis, Tongue cancer, Survival analysis, Lymph node metastasis

\section{Background}

Tongue cancer is one of the most common cancers in the oral cavity, most of which are squamous cell carcinomas $[1,2]$. Lymph node metastasis tends to occur during the early stage of tongue cancer, and it is one of the important factors leading to poor prognosis [3]. Lymph node metastasis during tumor progression involves the

\footnotetext{
*Correspondence: lumengmeng19810428@126.com; daiqunkqyy@163.com

${ }^{1}$ The Affiliated Stomatological Hospital of Nanchang University, The Key Laboratory of Oral Biomedicine, 49 Fuzhou Road, Nanchang 330000,

China

2 Department of Oral and Maxillofacial Surgery, Shanghai Stomatological Hospital, Fudan University, 1258 Fuxin Zhong Road, Shanghai 200031, China
}

deregulation of multiple gene and signaling pathways. In this process cancer cells separate from the primary tumor site and penetrate the basement membrane by degrading the extracellular matrix and then permeate into the lymphatic vessels. Many signaling pathways are associated with this process, such as PI3K-AKT, hedgehog, and NF-kB signaling pathways [4-6], but the molecular process in tongue cancer remains unknown [7]. Therefore, finding key genes and pathways involved in lymph node metastasis and inhibiting the progression of tongue cancer at the molecular level is expected to improve the prognosis of patients with tongue cancer.

In recent years, with the development of bioinformatics, genomic sequencing, gene chip, and data mining

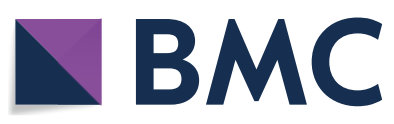

(c) The Author(s) 2019. This article is distributed under the terms of the Creative Commons Attribution 4.0 International License (http://creativecommons.org/licenses/by/4.0/), which permits unrestricted use, distribution, and reproduction in any medium, provided you give appropriate credit to the original author(s) and the source, provide a link to the Creative Commons license, and indicate if changes were made. The Creative Commons Public Domain Dedication waiver (http://creativecommons.org/ publicdomain/zero/1.0/) applies to the data made available in this article, unless otherwise stated. 
have played an important role in personalized medicine $[8,9]$. Using bioinformatic techniques to mine and analyze microarrays and sequencing datasets can provide a new direction for studies on tumor development and progression. GEO (Gene Expression Omnibus) is a public database that contains microarray, sequencing and high-throughput functional genomic datasets [10]. TCGA (The Cancer Genome Atlas) provides genomic sequencing datasets and clinical data for 33 types of cancer [11]. Both GEO and TCGA have made lasting contributions to understanding the mechanisms of cancer development and progression and have identified novel targets for cancer therapy [12].

In this study, we identified that IER3 was an important gene associated with lymph node metastasis and prognosis in tongue cancer patients by analyzing the GSE2280 RNA microarray dataset and the TCGA tongue cancer RNA-seq dataset. We then investigated the roles of IER3 in tongue cancer cells by performing proliferation, migration and invasion assays, and we also demonstrated that IER3 could promote lymphangiogenesis in vitro.

\section{Methods and materials \\ Data source}

The dataset of primary tongue cancer tissues and metastatic lymph node tissues was downloaded from the GEO database. The accession number was GSE2280 [13]. The microarray data of GSE2280 was based on GPL96 ([HGU133A] Affymetrix Human Genome U133A Array). The preprocessed level 3 RNA-seq data and clinical information of 148 tongue cancer patients with detailed followup information were downloaded from the TCGA data portal.

\section{Data analysis}

The $t$ scores of hallmark gene sets larger than 1 were obtained by using the "GSVA" package (Gene Set Variation Analysis) in R language [14], and hallmark gene sets are coherently expressed signatures derived by aggregating many MSigDB gene sets to represent welldefined biological states or processes [15]. Differentially expressed genes with $\log \mathrm{FC}>1$ and $\mathrm{P}$ value $<0.05$ were obtained by using the "limma" package [16]. The "pheatmap" package in R software was used to visualize differentially expressed genes. The Benjamini and Hochberg method was used to calculate the P value. Cytoscape software was used to establish a gene coexpression network and determine hub genes. The "survival" and "survminer" packages were used to perform survival analysis and create survival curves.

\section{Cell culture}

Human oral tongue squamous carcinoma cell line Tca8113 and human lymphatic endothelial cells (LECs) were purchased from the Tumor Cell Bank of the Chinese Academy of Medical Science (Beijing, China). Tongue cancer cell lines were cultured in DMEM (Gibco, USA) with $10 \%$ fetal bovine serum (FBS, HyClone, USA) and $1 \%$ penicillin/streptomycin at $37^{\circ} \mathrm{C}$ with $5 \% \mathrm{CO}_{2}$. LECs were maintained in RPMI-1640 medium (Gibco, USA) with 10\% fetal bovine serum and $1 \%$ penicillin/streptomycin.

\section{Migration and invasion assays}

Cell migration and invasion assays were performed using a 24-well transwell chamber with an $8 \mu \mathrm{m}$ pore size polycarbonate membrane (Corning, Corning, NY, USA). For the invasion assay, the membranes of 24-well transwell chambers were pretreated with Matrigel (BD Biosciences, USA) in $200 \mu \mathrm{l}$ serum-free medium. Briefly, $2 \times 10^{5}$ control or transfected cells were seeded on the chamber, and $500 \mu \mathrm{l}$ culture medium with $10 \%$ FBS was added to the lower chamber. After incubating for $24 \mathrm{~h}$, the cells were fixed with $5 \%$ paraformaldehyde for $20 \mathrm{~min}$. After removing the cells on the upper surface of the chamber, the cells on the lower surface of the chamber were stained with $0.1 \%$ crystal violet. The number of cells was counted in five random fields, and photos were taken under a microscope (Olympus).

\section{Cell proliferation assay}

The cell proliferation assay was performed using the Cell Counting Kit-8 (CCK-8, Dojindo, Japan). A total of $4 \times 10^{3}$ control or transfected cells were seeded into 96-well plates. After incubating for $24 \mathrm{~h}, 48 \mathrm{~h}$ and $72 \mathrm{~h}$, $10 \mu \mathrm{l} \mathrm{CCK-8} \mathrm{solution} \mathrm{was} \mathrm{added} \mathrm{to} \mathrm{each} \mathrm{well} \mathrm{and} \mathrm{then}$ incubated for another $4 \mathrm{~h}$ at $37^{\circ} \mathrm{C}$. The $450 \mathrm{~nm}$ OD value was measured using a microplate spectrophotometer (BioTek, USA).

\section{Colony formation assay}

A total of $5 \times 10^{2}$ control or transfected cells were seeded in 6-well plates. The culture medium was refreshed every 4 days. After incubating for 14 days, colonies were fixed with $5 \%$ paraformaldehyde for $20 \mathrm{~min}$ and then stained with $0.1 \%$ crystal violet solution for $15 \mathrm{~min}$. The number of colonies was counted, and the cells were photographed with a camera.

\section{Enzyme-linked immunosorbent assay (ELISA)}

ELISA was used to determine the effects of si-IER3 on the secretion of VEGF-C in tongue cancer cells. 
The cell culture medium of Tca-8113 and si-IER3treated Tca-8113 cells was collected, and a VEGF-C ELISA kit (eBioscience, Thermo Fisher, USA) was used to examine VEGF-C expression according to the manufacturer's instructions.

\section{RNA interference}

IER3 siRNA was purchased from RiboBio (Guangzhou). Cells were transfected with either $5 \mu \mathrm{M}$ si-IER3-1 or siIER3-2 using Lipofectamine 3000 (Invitrogen, USA) according to the manufacturer's protocols, control group in this study was only transfected with Lipofectamine 3000 without Si-RNA. The expression of IER3 was evaluated by Western blot and q-PCR. The si-IER3-1 sequence was $5^{\prime}$-AGCAGCAGAAAGAGAAGAAGCCtt- $3^{\prime}$. The siIER3-2 sequence was 5'-GGAAGGAGAGCGUCGUUA A- $3^{\prime}$.

\section{RNA isolation and quantitative real-time polymerase chain} reaction

Total RNA was extracted from cells using TRIzol (Takara, Japan) according to the manufacturer's instructions. A total of $500 \mathrm{ng}$ of total RNA was used for cDNA synthesis by using a reverse transcription kit (Takara, Japan). qRT-PCR was performed using a 7500 HT Fast Real-Time PCR System (Applied Biosystems, USA). mRNA expression was normalized with GAPDH, and data are presented as an expression fold change using the $2-\Delta \Delta \mathrm{Ct}$ method. Primers were purchased from Sangon Biotech (China). The IER3 primer sequences were forward $5^{\prime}$-TCCTGTTTTGTCTCCCCTTACG- $3^{\prime}$ and reverse $5^{\prime}$-TCAGGATCTGGCAGAAGACGAT- $3^{\prime}$. The VEGF-C primer sequences were forward $5^{\prime}$-CAGTTA CGGTCTGTGTCCAGTGTAG- $3^{\prime}$ and reverse $5^{\prime}$-GGA CACACATGGAGGTTTAAAGAAG- $3^{\prime}$. The VEgF-D primer sequences were forward $5^{\prime}$-TGGGTCATCTTC TCGCGGTT- $3^{\prime}$ and reverse $5^{\prime}$-GATGATGATATCGCC GCGCT- ${ }^{\prime}$.

\section{Tube formation assay}

The tube formation assay was performed using $\mu$-Plate Angiogenesis 96 Well according to the manufacturer's instructions and a previous study. Briefly, the Matrigel (BD Biosciences, USA) coat formed a solid structure on the bottom of a 96-well plate. Control or transfected LECs were suspended in culture media and seeded onto Matrigel-pretreated 96-well plates. After incubation, LECs were observed for tube formation under a lightfield microscope. The tubes were imaged, and the number of tubes was counted.

\section{Western blot analysis}

Total protein was extracted from cells with lysis buffer (KeyGEN BioTECH, China). Total protein samples were separated by SDS-PAGE (10-15\%) and transferred to a PVDF membrane. After blocking with 5\% defatted milk for $1 \mathrm{~h}$, the membrane was incubated with primary antibodies overnight at $4{ }^{\circ} \mathrm{C}$ and then further incubated with HRP-conjugated secondary antibody for $1 \mathrm{~h}$ at room temperature. Protein bands were detected using chemiluminescence (KeyGEN BioTECH, China).

\section{Statistical analysis}

All data are presented as the mean \pm standard deviation (SD). Student's t-test was used to calculate the P-value when comparing two groups. A one-way analysis of variance (ANOVA) was used when comparing more than two groups. SPSS 18.0 was used to analyze all data. In all cases, two-sided P-values $<0.05$ were considered statistically significant.

\section{Results}

Analysis of hallmark gene sets involved in lymph node metastasis

Hallmark gene sets consist of fifty sets that represent over four thousands original overlapping gene sets from v4.0 MSigDB collections C1 to C6, and provide accurate results when used with enrichment analysis methods in cancer [15]. We only found one dataset (GSE2280) of tongue cancer that compared the gene expression of metastatic lymph node with primary cancer tissues from GEO datasets. Then, we analyzed the changes in hallmark gene sets in metastatic lymph nodes through GSVA. As shown in Fig. 1, we found that six hallmark gene sets were upregulated (t score $>1$ ), eleven hallmark gene sets were downregulated ( $t$ score $<-1$ ), and in total the changed hallmark gene sets contained 1839 genes.

Screening of hub genes involved in lymph node metastasis To further investigate the hub genes from these hallmark gene sets that were involved in lymph node metastasis, we analyzed the change in expression of the 1839 genes in metastatic lymph nodes and primary tumors (Additional file 1: Table S1). As shown in Fig. 2a, in a total of 1839 genes, 76 genes were deregulated, of which 21 genes were upregulated and 55 genes were downregulated. Most of these deregulated genes were enriched in MYOGENESIS, KRAS_SIGNALING_UP, HYPOXIA, G2M_CHECKPOINT, and E2F_TARGETS (Table 1). Then, a molecular network of the deregulated genes was established, and the top 10 deregulation hub genes were identified by Cytoscape 


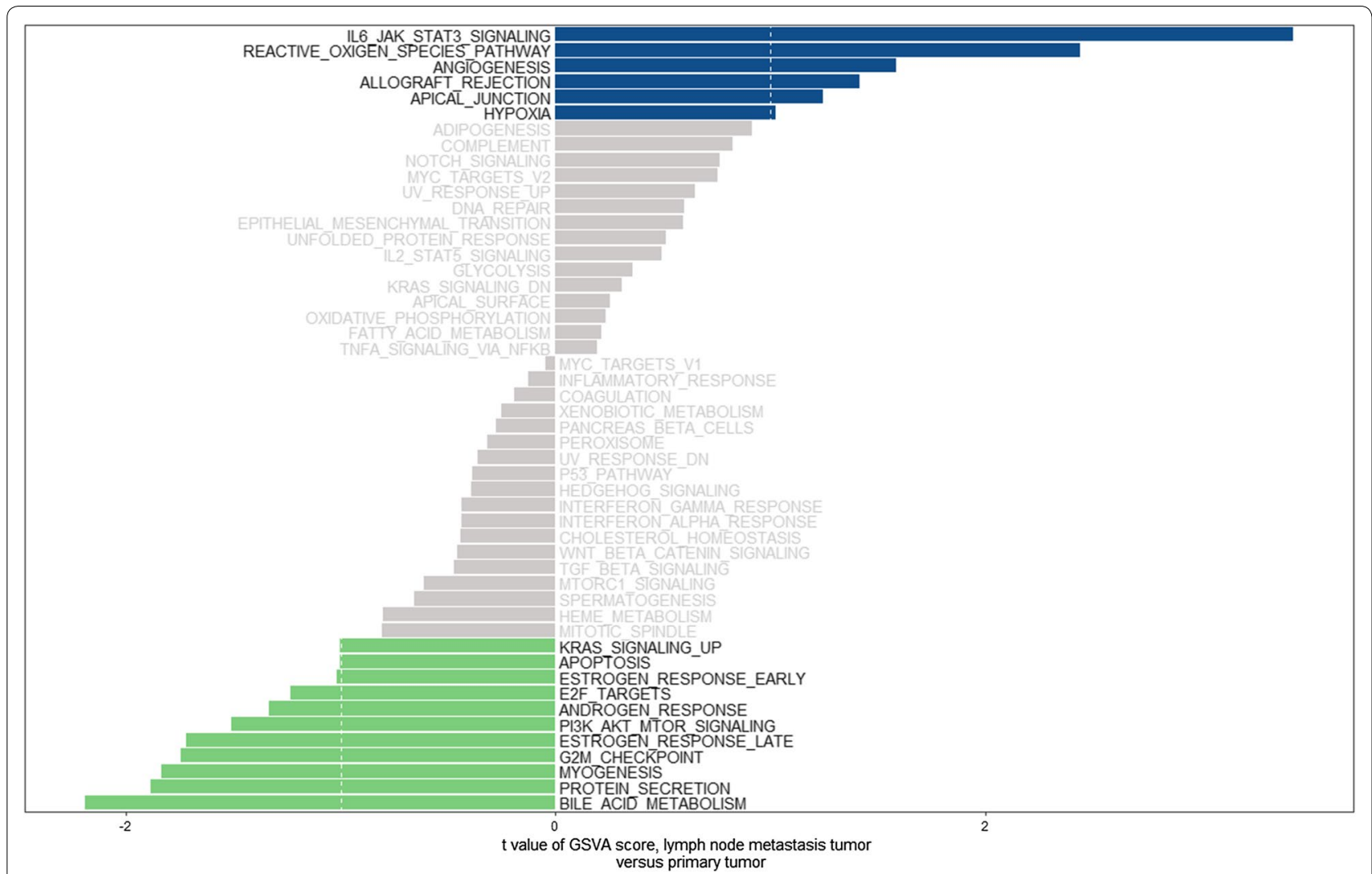

Fig. 1 The changed Hallmark gene sets of metastatic lymph node versus primary tumor. A t value $>1$ or $<-1$ represents statistically significant changes

(Fig. 2b), of which only the expression of IER3 was upregulated $(\log F C=1.38, P=0.015)$, while the other 9 genes were downregulated (Table 2).

\section{Subsequent screening and validation of the hub genes using TCGA data}

To investigate whether candidate genes are involved in lymph node metastasis and the prognosis of tongue cancer patients, we analyzed TCGA tongue cancer data for validation and for further screening of functional genes. Overall survival and lymph node metastasis data of TCGA tongue cancer patients were extracted from the TCGA-HNSC dataset. Based on these data, we found that patients with high expression of IER3 had poor prognosis (Fig. 3a), and those with high expression of IOD2 had better prognosis (Additional file 2: Figure S1); however, the other candidate genes had no significant association with overall survival (Additional file 2: Figure S1 and Table 3). More importantly, tongue cancer patients with lymph node metastasis showed significantly higher expression of IER3, and high expression of IER3 showed better recurrence-free survival (Fig. 3b, c).

\section{Knockdown of IER3 inhibits the proliferation, migration and invasion of Tca-8113 cells}

As shown above, we found that IER3 might be a key gene that promotes the progression and lymph node metastasis of tongue cancer. We examined the protein expression of IER3 in 2 tongue cancer cell lines and 2 oral squamous cell carcinoma cell lines, and the results showed that all 4 oral cancer cell lines expressed IER3 (Fig. 4a). We knocked down the expression of IER3 in Tca-8113 cells by transfecting specific siRNA, and the validation results are shown in Fig. 4b, c. The results of proliferation and colony formation assays suggested that knocking down IER 3 could significantly suppress cell proliferation in Tca8113 and SCC-25 cells (Fig. 4d, e). Furthermore, Fig. 5 shows that knockdown of IER3 inhibited the migration and invasion of Tca-8113 and SCC-25 cells (Fig. 5a-d).

\section{Knockdown of IER3 in Tca-8113 cells inhibits lymphangiogenesis and migration of LECs}

VEGF-C and VEGF-D have been identified as cytokines that promote lymphangiogenesis and lymph node metastasis in cancer. We found that knockdown of IER3 

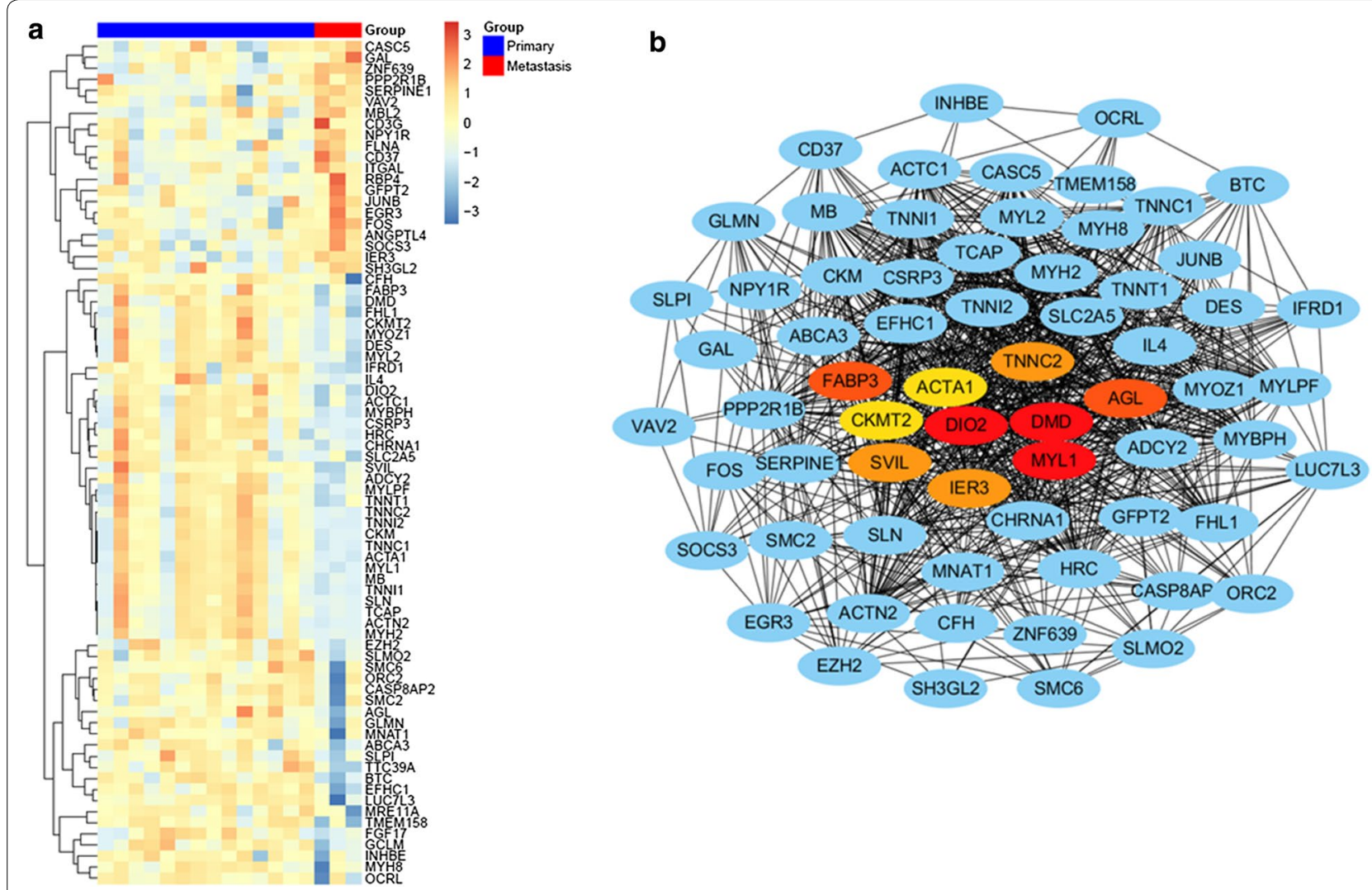

Fig. 2 Screening of hub genes involved with lymph node metastasis. a Heatmap of the deregulated genes in metastatic lymph node versus primary tumor. b Gene coexpression network of the 76 differentially expressed genes. The red, yellow and orange ovals represent ten candidate hub genes in the network

decreased the mRNA expression of VEGF-C but not VEGF-D in Tca-8113 cells (Fig. 6a, b), and Western blot results showed that the protein expression of VEGF-C also decreased (Fig. 6c). In addition, the results of the ELISA assay showed that the secretion of VEGF-C in Tca-8113 cells was decreased when IER3 was knocked down (Fig. 6d). We performed migration and tube formation assays in LECs using the culture supernatants of Tca-8113 and si-IER3-transfected Tca-8113 cells. The results showed that the number of migrated cells in the Tca-8113 group was significantly higher than that in the control and si-IER3 groups (Fig. 7a, c), and the tube number in the Tca-8113 group was also higher than that in the control and si-IER3 groups (Fig. 7b, d).

\section{Discussion}

Tongue cancer is one of the most common cancers in oral squamous cell carcinoma, and the development of oral cancer is a multi-step process involving a variety of molecular and genomic deregulation steps. Lymph node metastasis is an important factor affecting the prognosis of tongue cancer, and the effective molecular targets remain unknown. In this study, we identified the hallmark gene sets and hub genes involved in the lymph node metastasis of tongue cancer. Moreover, based on TCGA data, we found that IER3 was the key gene that might promote lymph node metastasis and a potential marker of prognosis for tongue cancer patients. We validated the function of IER3 in tongue cancer cells, and the results showed that knocking down IER3 significantly decreased the proliferation, migration and invasion of Tca-8113 cells and decreased the secretion of VEGF-C, which reduced the tube formation and migration of LECs.

GSVA is a method that builds on GSEA and is better than GSEA for characterizing pathways from data obtained from both microarray and RNA-seq assays. GSVA is widely used in bioinformatic analysis of different tumors for investigating the molecular mechanism 
Table $1 \mathrm{~A}$ total of $\mathbf{7 6}$ deregulated genes were identified in 1839 primary tumor versus metastatic lymph node genes

\begin{tabular}{|c|c|c|c|}
\hline Gene symbol & $\log \mathrm{FC}$ & $P$ value & Hallmark \\
\hline MBL2 & 1.836355 & 0.016409 & ALLOGRAFT_REJECTION \\
\hline ITGAL & 1.09615 & 0.029208 & ALLOGRAFT_REJECTION \\
\hline CD3G & 1.084988 & 0.023672 & ALLOGRAFT_REJECTION \\
\hline FLNA & 1.003019 & 0.037726 & ALLOGRAFT_REJECTION \\
\hline GLMN & -1.11045 & 0.044154 & ALLOGRAFT_REJECTION \\
\hline VAV2 & 1.222483 & 0.037575 & ANGIOGENESIS, APICAL_JUNCTION \\
\hline ACTA1 & -4.81243 & 0.007734 & APICAL_JUNCTION \\
\hline $\mathrm{EFHC1}$ & -1.33106 & 0.001242 & BILE_ACID_METABOLISM \\
\hline $\mathrm{DIO} 2$ & -1.48714 & 0.003577 & BILE_ACID_METABOLISM \\
\hline SMC6 & -1.01378 & 0.036294 & E2F_TARGETS \\
\hline ORC2 & -1.02171 & 0.039099 & E2F_TARGETS \\
\hline LUC7L3 & -1.21609 & 0.002313 & E2F_TARGETS \\
\hline MRE11A & -1.23003 & 0.045764 & E2F_TARGETS \\
\hline ABCA3 & -1.4387 & 0.009719 & ESTROGEN_RESPONSE_EARLY, ESTROGEN_RESPONSE_LATE \\
\hline TTC39A & -1.90927 & 0.002779 & ESTROGEN_RESPONSE_EARLY \\
\hline NPY1R & 2.020464 & 0.03779 & ESTROGEN_RESPONSE_LATE, ESTROGEN_RESPONSE_EARLY \\
\hline GAL & 1.468347 & 0.023249 & ESTROGEN_RESPONSE_LATE \\
\hline EGR3 & 1.177485 & 0.036595 & $\begin{array}{l}\text { ESTROGEN_RESPONSE_LATE, ESTROGEN_RESPONSE_EARLY, } \\
\text { KRAS_SIGNALING_UP }\end{array}$ \\
\hline CASC5 & 1.897606 & 0.036429 & G2M_CHECKPOINT \\
\hline CASP8AP2 & -1.09086 & 0.016979 & G2M_CHECKPOINT \\
\hline $\mathrm{EZH} 2$ & -1.18721 & 0.018306 & G2M_CHECKPOINT, E2F_TARGETS \\
\hline MNAT1 & -1.27108 & 0.027402 & G2M_CHECKPOINT \\
\hline SMC2 & -1.33982 & 0.04864 & G2M_CHECKPOINT \\
\hline SERPINE1 & 1.733088 & 0.021088 & HYPOXIA \\
\hline FOS & 1.543577 & 0.005153 & HYPOXIA \\
\hline IER3 & 1.387516 & 0.015602 & HYPOXIA, APOPTOSIS \\
\hline ANGPTL4 & 1.261686 & 0.018984 & HYPOXIA, KRAS_SIGNALING_UP, ADIPOGENESIS \\
\hline SLC2A5 & -2.14747 & 0.047968 & HYPOXIA \\
\hline SOCS3 & 1.72843 & 0.019538 & IL6_JAK_STAT3_SIGNALING \\
\hline INHBE & -1.17054 & 0.031406 & IL6_JAK_STAT4_SIGNALING \\
\hline ZNF639 & 2.511151 & 0.001222 & KRAS_SIGNALING_UP \\
\hline RBP4 & 2.229625 & 0.033045 & KRAS_SIGNALING_UP \\
\hline CD37 & 1.547497 & 0.019287 & KRAS_SIGNALING_UP \\
\hline GFPT2 & 1.031506 & 0.042835 & KRAS_SIGNALING_UP \\
\hline SLMO2 & -1.00092 & 0.02985 & KRAS_SIGNALING_UP \\
\hline $\mathrm{CFH}$ & -1.6692 & 0.040962 & KRAS_SIGNALING_UP \\
\hline SLPI & -1.67431 & 0.014661 & KRAS_SIGNALING_UP \\
\hline BTC & -2.14105 & 0.00012 & KRAS_SIGNALING_UP \\
\hline TMEM158 & -2.44114 & 0.000586 & KRAS_SIGNALING_UP \\
\hline SVIL & -1.15372 & 0.004237 & MYOGENESIS \\
\hline IFRD1 & -1.29777 & 0.000871 & MYOGENESIS \\
\hline AGL & -1.34563 & 0.006822 & MYOGENESIS \\
\hline FABP3 & -1.39162 & 0.018123 & MYOGENESIS \\
\hline CKMT2 & -1.43326 & 0.0434 & MYOGENESIS \\
\hline MYH8 & -1.48548 & 0.015832 & MYOGENESIS \\
\hline DMD & -1.53731 & 0.006311 & MYOGENESIS, G2M_CHECKPOINT \\
\hline MYOZ1 & -1.74048 & 0.041979 & MYOGENESIS \\
\hline CHRNA1 & -1.8081 & 0.017455 & MYOGENESIS \\
\hline $\mathrm{HRC}$ & -2.02106 & 0.038865 & MYOGENESIS \\
\hline
\end{tabular}


Table 1 (continued)

\begin{tabular}{|c|c|c|c|}
\hline Gene symbol & $\log \mathrm{FC}$ & $P$ value & Hallmark \\
\hline FHL1 & -2.03688 & 0.035865 & MYOGENESIS \\
\hline SLN & -2.11469 & 0.041452 & MYOGENESIS \\
\hline ACTC1 & -2.90968 & 0.011697 & MYOGENESIS \\
\hline DES & -3.03388 & 0.031095 & MYOGENESIS \\
\hline MYBPH & -3.21711 & 0.003079 & MYOGENESIS \\
\hline TNNT1 & -3.34517 & 0.024009 & MYOGENESIS \\
\hline MYH2 & -3.3503 & 0.042848 & MYOGENESIS \\
\hline $\mathrm{MB}$ & -3.35038 & 0.010556 & MYOGENESIS \\
\hline TCAP & -3.38871 & 0.029364 & MYOGENESIS \\
\hline MYLPF & -3.50266 & 0.020669 & MYOGENESIS \\
\hline TNNC2 & -3.52148 & 0.020435 & MYOGENESIS \\
\hline MYL2 & -3.59938 & 0.011658 & MYOGENESIS \\
\hline ACTN2 & -4.31463 & 0.01354 & MYOGENESIS \\
\hline TNNI2 & -4.42965 & 0.011318 & MYOGENESIS \\
\hline MYL1 & -4.54843 & 0.006995 & MYOGENESIS \\
\hline CKM & -4.71858 & 0.011289 & MYOGENESIS \\
\hline TNNI1 & -4.77636 & 0.0062 & MYOGENESIS \\
\hline TNNC1 & -5.64825 & 0.007417 & MYOGENESIS \\
\hline CSRP3 & -5.77325 & 0.012999 & MYOGENESIS \\
\hline PPP2R1B & 1.171797 & 0.042423 & PI3K_AKT_MTOR_SIGNALING \\
\hline FGF17 & -1.34021 & 0.032683 & PI3K_AKT_MTOR_SIGNALING \\
\hline IL4 & -1.52042 & 0.040415 & PI5K_AKT_MTOR_SIGNALING, IL6_JAK_STAT3_SIGNALING \\
\hline ADCY2 & -1.91434 & 0.025949 & PI6K_AKT_MTOR_SIGNALING \\
\hline SH3GL2 & 1.508413 & 0.042024 & PROTEIN_SECRETION \\
\hline OCRL & -1.09672 & 0.008807 & PROTEIN_SECRETION \\
\hline JUNB & 1.047607 & 0.019591 & REACTIVE_OXIGEN_SPECIES_PATHWAY \\
\hline GCLM & -1.71176 & 0.003821 & REACTIVE_OXIGEN_SPECIES_PATHWAY, BILE_ACID_METABOLISM \\
\hline
\end{tabular}

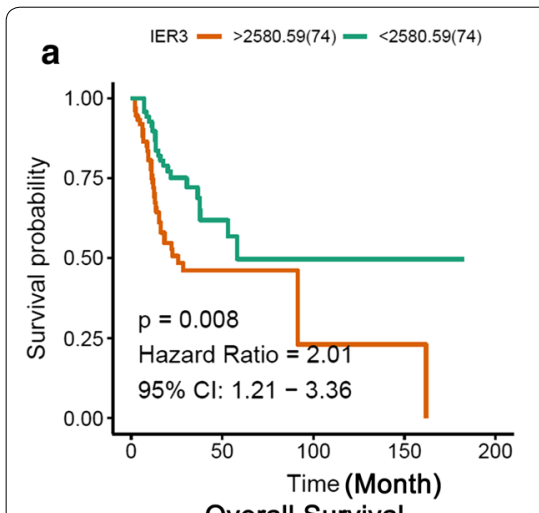

Overall Survival

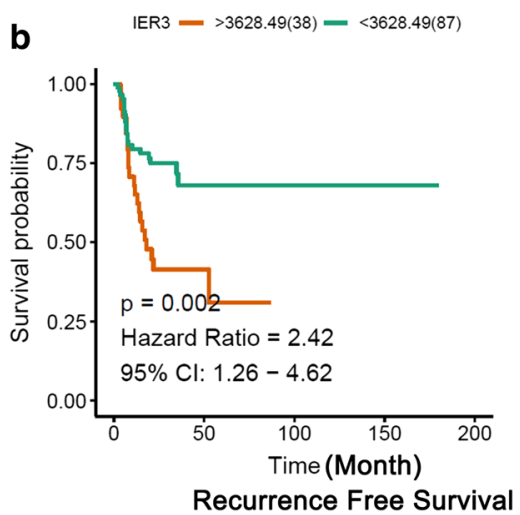

C

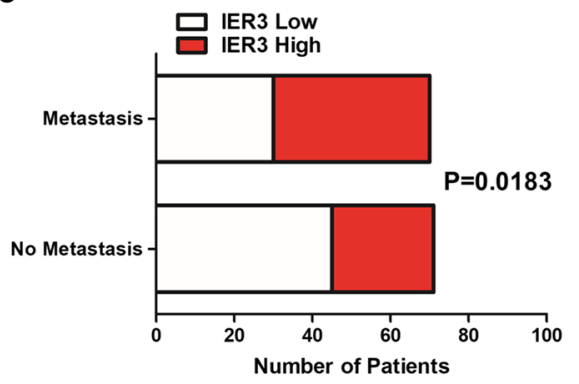

Recurrence Free Survival

Fig. 3 Expression of IER3 is correlated with overall survival and lymph node metastasis. a Overall survival of 148 tongue cancer patients (TCGA data) analyzed by the Kaplan-Meier method and log-rank test according to IER3 expression. b Recurrence-free survival of 148 tongue cancer patients (TCGA data) analyzed by the Kaplan-Meier method and log-rank test according to IER3 expression. c Correlations between the expression of IER3 and lymph node metastasis in tongue cancer patients $(n=141)$ 
Table 2 Top 10 hubgenes in $\mathbf{7 6}$ deregulated genes

\begin{tabular}{llcll}
\hline Gene & logFC & AveExpr & t & P value \\
\hline ACTA1 & -4.81243 & 8.399569 & -2.99088 & 0.007734 \\
AGL & -1.34563 & 7.828326 & -3.04814 & 0.006822 \\
CKMT2 & -1.43326 & 6.654878 & -2.16996 & 0.0434 \\
DIO2 & -1.48714 & 7.679014 & -3.34018 & 0.003577 \\
DMD & -1.53731 & 6.485171 & -3.08362 & 0.006311 \\
FABP3 & -1.39162 & 5.339507 & -2.59505 & 0.018123 \\
IER3 & 1.387516 & 10.10791 & 2.665727 & 0.015602 \\
MYL1 & -4.54843 & 8.410023 & -3.03672 & 0.006995 \\
SVIL & -1.15372 & 9.014868 & -3.26402 & 0.004237 \\
TNNC2 & -3.52148 & 6.850598 & -2.53798 & 0.020435 \\
\hline
\end{tabular}

of cancer development and progression [17, 18]. Based on GSVA, we identified 17 hallmark gene sets involved in lymph node metastasis, of which the upregulated hallmark gene sets IL6_JAK_STAT3_SIGNALING, REACTIVE_OXIGEN_SPECIES_PATHWAY,ANGIOGENESIS, HYPOXIA and APICAL_JUNCTION were reportedly associated with lymph node metastasis in cancer [19-22]. However, the downregulated hallmark gene sets were also related to lymph node metastasis, such as KRAS_SIGNALING and PI3 K_AKT_MTOR_SIGNALING [23, 24], which indicated that the mechanism of lymph node metastasis is complicated and involves various activated and inactivated signaling pathways. In our

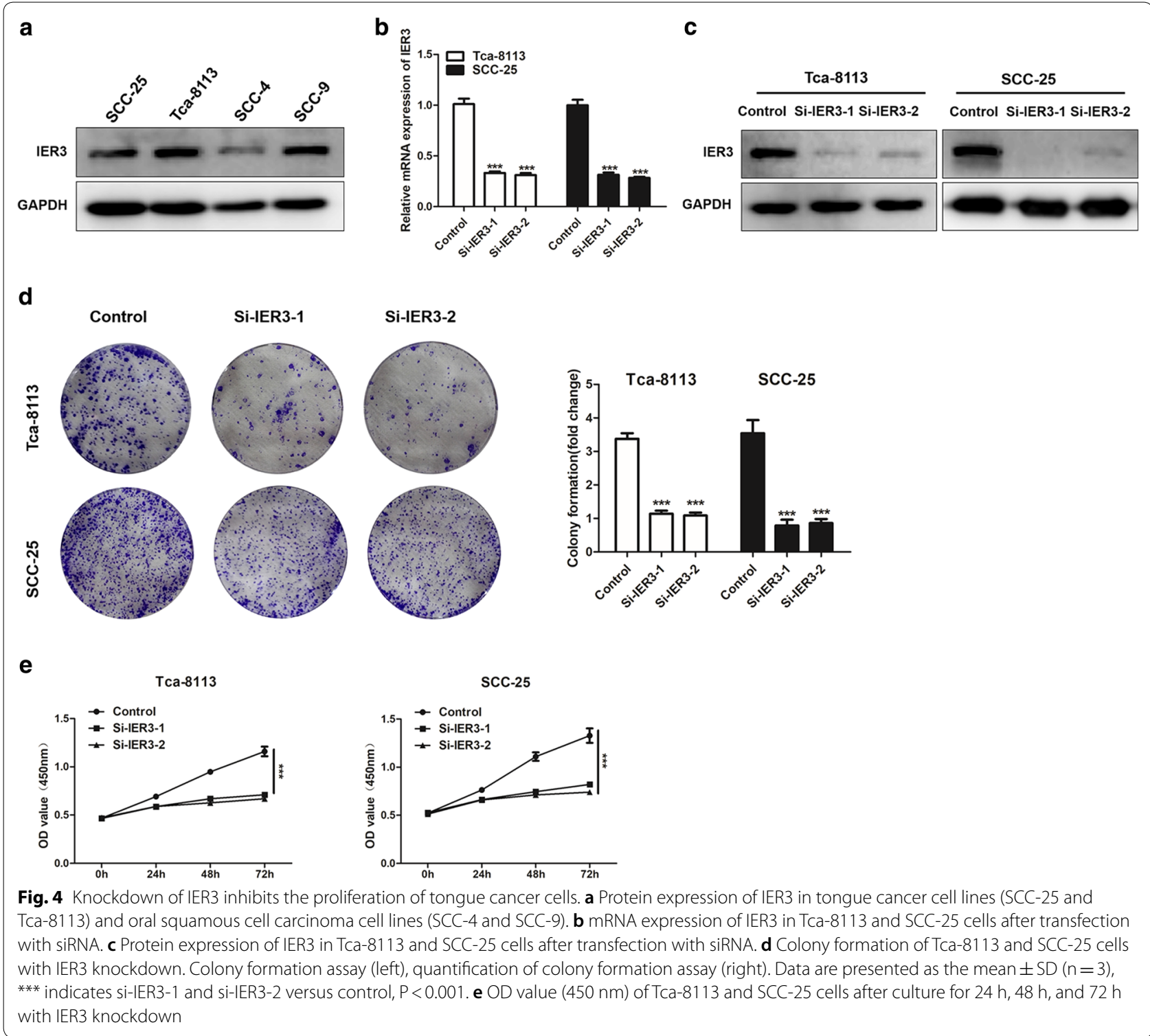




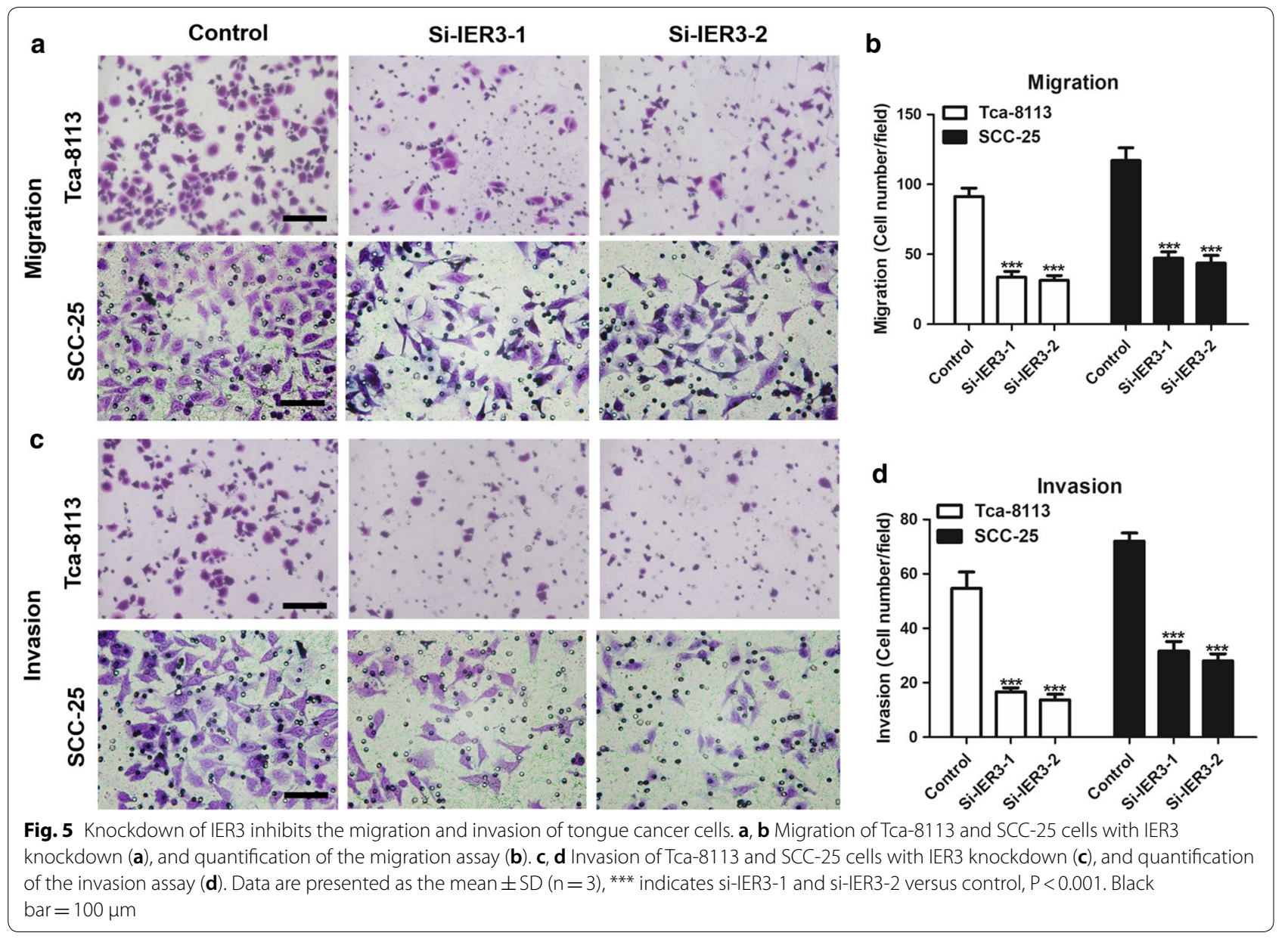

study, each deregulated hallmark gene set contained many genes, not all of which were deregulated. Therefore, we analyzed the expression of all 1839 genes in the identified hallmark gene sets and found that 76 genes were deregulated and were mostly enriched in MYOGENESIS, ANGIOGENESIS, HYPOXIA, KRAS_SIGNALING_UP, and G2M_CHECKPOINT, of which MYOGENESIS, G2M_CHECKPOINT, and KRAS_SIGNALING_UP were downregulated in metastatic lymph nodes, and ANGIOGENESIS and HYPOXIA were upregulated. It has been reported that HYPOXIA and ANGIOGENESIS can promote lymph node metastasis in cancer $[25,26]$. MYOGENESIS is associated with the formation of muscle tissue during embryonic development [27], and we speculate that its upregulation in metastatic lymph nodes might be associated with the differences in cells in primary and metastatic tumor tissues, such as muscles and fibroblasts, because tissue heterogeneity affects the accuracy of microarray and sequencing data [28]. The downregulation of G2M_CHECKPOINT and KRAS_SIGNALING_UP might contribute to the low proliferation ability of metastatic cancer cells [29]. Finally, we found that IER3 might be the key gene that could promote lymph node metastasis, and the results of validation using TCGA data could demonstrate it.

IER3, also known as IEX-1, p22/PRG1, Dif-2, or gly96, belongs to the early response gene family, which is involved in cell differentiation and cell proliferation [30]. IER3 is highly expressed in a variety of tumors and is associated with poor prognosis, such as bladder cancer, pancreatic cancer, breast cancer, melanoma, etc. [30, 31]. However, no studies have reported its relationship with tongue cancer. Our study found that high expression of IER3 was associated with poor prognosis and lymph node metastasis in patients with tongue cancer, suggesting that IER3 might have a cancer-promoting effect in tongue cancer.

We found that IER3 could promote the proliferation, invasion and migration of tongue cancer cells in vitro. Previous studies have reported that IER3 is involved in the activation of the PI3 K/AKT and MAPK/ERK signaling pathways [32], which promotes tumor progression, also suggesting that IER3 plays a role in promoting tongue cancer, but further in vivo evidence 


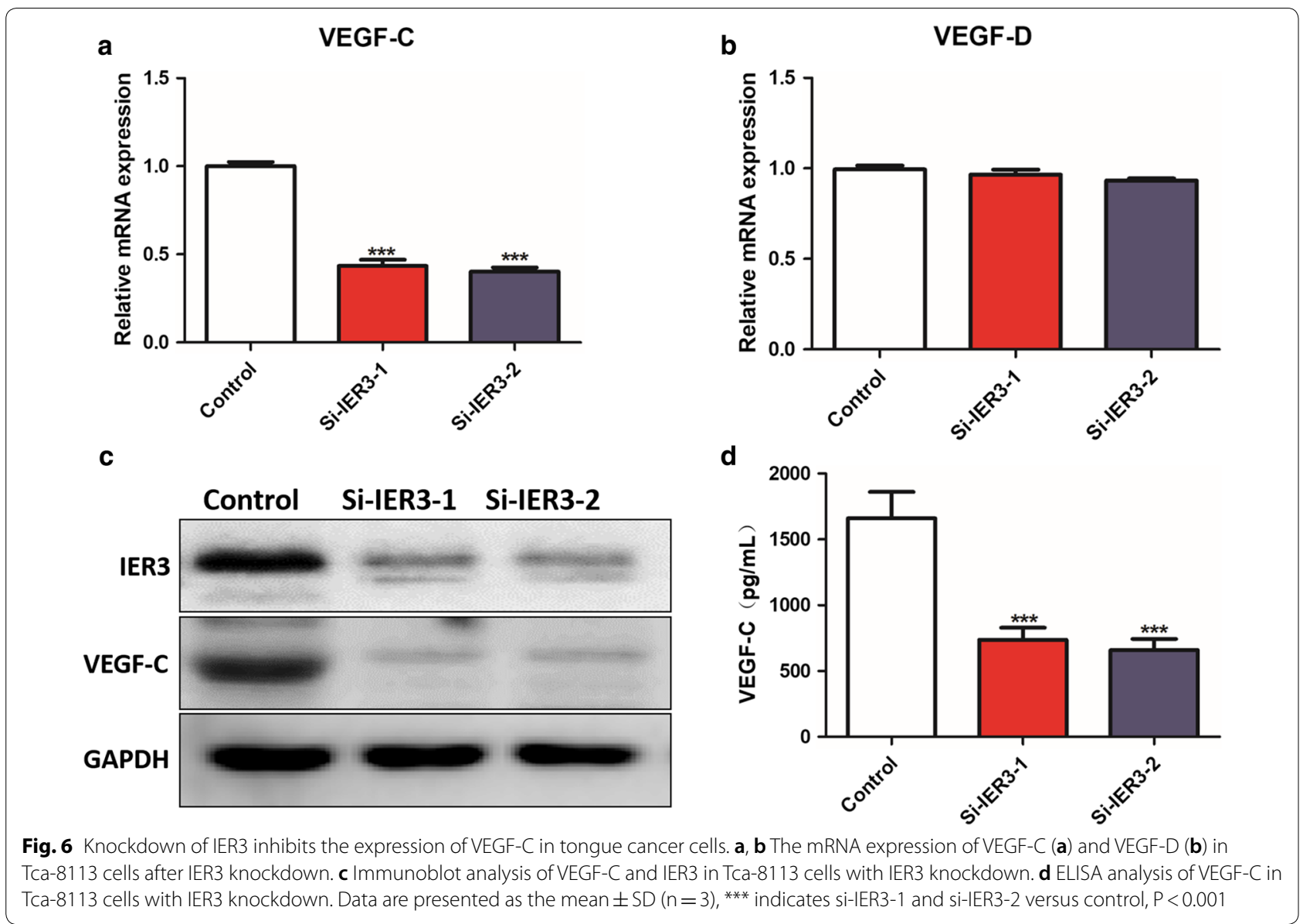

is needed. More importantly, we found that knocking down IER3 reduced the secretion of VEGF-C from tongue cancer cells, which could promote the formation of lymphatic vessels and lymph node metastasis $[33,34]$. Our studies demonstrated that tongue cancer cell supernatant increased the tube formation of LECs, while the supernatant of IER3-downregulated tongue cancer cells could not, which indicated that IER3 could promote lymphangiogenesis by promoting the secretion of VEGF-C, and lymph node metastasis in tongue cancer was associated with this process. The activation of the PI3 K/AKT and JAK2/STAT3 signaling pathways could promote the secretion of VEGF-C in cancer cells
[35, 36], suggesting that IER3 might induce the generation of VEGF-C in tongue cancer cells, but the specific molecular mechanism needs to be identified in further studies.

\section{Conclusions}

Our study identified the changed hallmark gene sets between metastatic lymph node and primary tongue cancer that are associated with lymph node metastasis in tongue cancer. More importantly, we found that the hub gene IER3 might predict prognosis tongue cancer, and our in vitro experiments demonstrated that IER3 might 


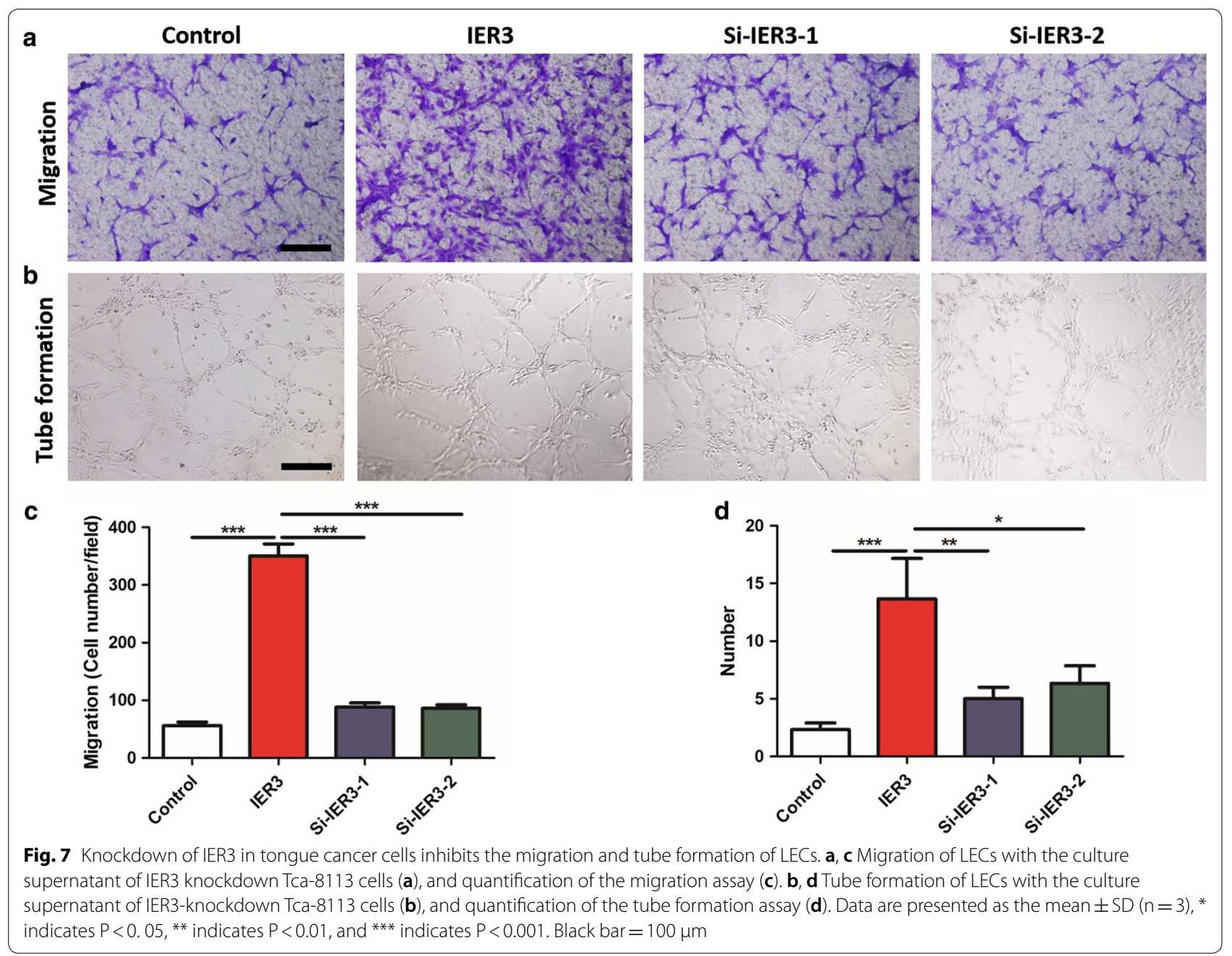

Table 3 Overall survival analysis of the top 10 hub genes of TCGA tongue cancer patients

\begin{tabular}{llll}
\hline Gene & Hazard ratio & $\mathbf{9 5 \%} \mathbf{C l}$ & P value \\
\hline IER3 & 2.01 & $1.21-3.36$ & 0.008 \\
DIO2 & 0.59 & $0.35-0.98$ & 0.045 \\
CKMT2 & 1.66 & $0.93-2.97$ & 0.055 \\
SVIL & 1.64 & $0.97-2.78$ & 0.086 \\
AGL & 1.5 & $0.89-2.54$ & 0.114 \\
FABP3 & 1.56 & $0.92-2.64$ & 0.082 \\
ACTA1 & 1.44 & $0.84-2.49$ & 0.164 \\
DMD & 0.75 & $0.45-1.25$ & 0.264 \\
MYL1 & 1.31 & $0.76-2.24$ & 0.308 \\
TNNC2 & 1.28 & $0.74-2.21$ & 0.397 \\
\hline
\end{tabular}

promote the progression and lymph node metastasis in tongue cancer, which might be a potential therapeutic target.

\section{Supplementary information}

Supplementary information accompanies this paper at https://doi. org/10.1186/s12935-019-1028-2.

Additional file 1: Table S1. New management strategies of oral tongue cancer in Bangladesh.

Additional file 2: Figure S1. Overall survival analysis of 9 hub genes of TCGA tongue cancer patients.
Abbreviations
GSVA: gene set variation analysis; GSEA: gene set enrichment analysis; TCGA: The Cancer Genome Atlas; OSCC: oral squamous cell carcinoma; OS: overall survival; RFS: recurrence-free survival; HR: hazard ratio.

\section{Acknowledgements}
Not applicable.

\section{Authors' contributions}

$\mathrm{QD}$ and $\mathrm{ML}$ designed the study. FX and YD performed the study. FX and YH analyzed the data and QD wrote the paper. All authors read and approved the final manuscript.

\section{Funding}

Not applicable. 


\section{Availability of data and materials}

The datasets used and/or analyzed during the study are available from the corresponding author on reasonable request.

\section{Ethics approval and consent to participate}

Not applicable.

\section{Consent for publication}

Not applicable.

\section{Competing interests}

The authors declare that they have no competing interests.

Received: 7 September 2019 Accepted: 11 November 2019

Published online: 21 November 2019

\section{References}

1. Sultana J, Bashar A, Molla MR. New management strategies of oral tongue cancer in Bangladesh. J Maxillofac Oral Surg. 2014;13(4):394-400.

2. Montero PH, Patel SG. Cancer of the oral cavity. Surg Oncol Clin N Am. 2015:24(3):491-508.

3. Biswas NK, Das C, Das S, Maitra A, Nair S, Gupta T, D'Cruz AK, Sarin R, Majumder PP. Lymph node metastasis in oral cancer is strongly associated with chromosomal instability and DNA repair defects. Int J Cancer. 2019;145(9):2568-79.

4. Yoo YA, Kang MH, Lee HJ, Kim BH, Park JK, Kim HK, Kim JS, Oh SC. Sonic hedgehog pathway promotes metastasis and lymphangiogenesis via activation of Akt, EMT, and MMP-9 pathway in gastric cancer. Cancer Res. 2011;71(22):7061-70.

5. Yuan TL, Cantley LC. PI3K pathway alterations in cancer: variations on a theme. Oncogene. 2008;27(41):5497-510.

6. Yan M, Xu Q, Zhang P, Zhou XJ, Zhang ZY, Chen WT. Correlation of NFkappaB signal pathway with tumor metastasis of human head and neck squamous cell carcinoma. BMC Cancer. 2010;10:437.

7. Irani S. Distant metastasis from oral cancer: a review and molecular biologic aspects. J Int Soc Prev Community Dent. 2016;6(4):265-71.

8. Ping Z, Siegal GP, Almeida JS, Schnitt SJ, Shen D. Mining genome sequencing data to identify the genomic features linked to breast cancer histopathology. J Pathol Inform. 2014;5(1):3.

9. Xia X. Bioinformatics and drug discovery. Curr Top Med Chem. 2017;17(15):1709-26.

10. Barrett T, Edgar R. Mining microarray data at NCBI's Gene Expression Omnibus (GEO). Methods Mol Biol. 2006:338:175-90.

11. Lee JS. Exploring cancer genomic data from the cancer genome atlas project. BMB Rep. 2016:49(11):607-11.

12. Doherty GJ, Petruzzelli M, Beddowes E, Ahmad SS, Caldas C, Gilbertson RJ. Cancer treatment in the genomic era. Annu Rev Biochem. 2019:88:247-80.

13. O'Donnell RK, Kupferman M, Wei SJ, Singhal S, Weber R, O'Malley B, Cheng Y, Putt M, Feldman M, Ziober B, et al. Gene expression signature predicts lymphatic metastasis in squamous cell carcinoma of the oral cavity. Oncogene. 2005;24(7):1244-51.

14. Hanzelmann S, Castelo R, Guinney J. GSVA: gene set variation analysis for microarray and RNA-seq data. BMC Bioinform. 2013;14:7.

15. Liberzon A, Birger C, Thorvaldsdottir H, Ghandi M, Mesirov JP, Tamayo P. The Molecular Signatures Database (MSigDB) hallmark gene set collection. Cell Syst. 2015;1(6):417-25.

16. Ritchie ME, Phipson B, Wu D, Hu Y, Law CW, Shi W, Smyth GK. limma powers differential expression analyses for RNA-sequencing and microarray studies. Nucleic Acids Res. 2015;43(7):e47.

17. Frost $\mathrm{HR}$, Amos $\mathrm{Cl}$. A multi-omics approach for identifying important pathways and genes in human cancer. BMC Bioinform. 2018;19(1):479.
18. Berger AC, Korkut A, Kanchi RS, Hegde AM, Lenoir W, Liu W, Liu Y, Fan H, Shen $\mathrm{H}$, Ravikumar $\mathrm{V}$, et al. A comprehensive pan-cancer molecular study of gynecologic and breast cancers. Cancer Cell. 2018;33(4):690-705.e699.

19. Chang Q, Bournazou E, Sansone P, Berishaj M, Gao SP, Daly L, Wels J, Theilen T, Granitto S, Zhang X, et al. The IL-6/JAK/Stat3 feed-forward loop drives tumorigenesis and metastasis. Neoplasia. 2013;15(7):848-62.

20. Wu H, Rahman HNA, Dong Y, Liu X, Lee Y, Wen A, To KH, Xiao L, Birsner $A E$, Bazinet $L$, et al. Epsin deficiency promotes lymphangiogenesis through regulation of VEGFR3 degradation in diabetes. J Clin Investig. 2018;128(9):4025-43.

21. Paduch $R$. The role of lymphangiogenesis and angiogenesis in tumor metastasis. Cell Oncol. 2016;39(5):397-410.

22. Naydenov NG, Ivanov Al. Adducins regulate remodeling of apical junctions in human epithelial cells. Mol Biol Cell. 2010;21(20):3506-17.

23. Coso S, Zeng Y, Opeskin K, Williams ED. Vascular endothelial growth factor receptor-3 directly interacts with phosphatidylinositol 3-kinase to regulate lymphangiogenesis. PLoS ONE. 2012;7(6):e39558.

24. Gore J, Imasuen-Williams IE, Conteh AM, Craven KE, Cheng M, Korc M. Combined targeting of TGF-beta, EGFR and HER2 suppresses lymphangiogenesis and metastasis in a pancreatic cancer model. Cancer Lett. 2016;379(1):143-53.

25. Ji RC. Hypoxia and lymphangiogenesis in tumor microenvironment and metastasis. Cancer Lett. 2014;346(1):6-16.

26. Gomes FG, Nedel F, Alves AM, Nor JE, Tarquinio SB. Tumor angiogenesis and lymphangiogenesis: tumor/endothelial crosstalk and cellular/microenvironmental signaling mechanisms. Life Sci. 2013;92(2):101-7.

27. Bentzinger CF, Wang YX, Rudnicki MA. Building muscle: molecular regulation of myogenesis. Cold Spring Harb Perspect Biol. 2012. https://doi. org/10.1101/cshperspect.a008342.

28. Bhattacharya S, Long D, Lyons-Weiler J. Overcoming confounded controls in the analysis of gene expression data from microarray experiments. Appl Bioinform. 2003;2(4):197-208.

29. Gupta SC, Kim JH, Prasad S, Aggarwal BB. Regulation of survival, proliferation, invasion, angiogenesis, and metastasis of tumor cells through modulation of inflammatory pathways by nutraceuticals. Cancer Metastasis Rev. 2010;29(3):405-34.

30. Garcia MN, Grasso D, Lopez-Millan MB, Hamidi T, Loncle C, Tomasini R, Lomberk G, Porteu F, Urrutia R, lovanna JL. IER3 supports KRASG12Ddependent pancreatic cancer development by sustaining ERK1/2 phosphorylation. J Clin Investig. 2014;124(11):4709-22.

31. Ye J, Zhang Y, Cai Z, Jiang M, Li B, Chen G, Zeng Y, Liang Y, Wu S, Wang Z, et al. Increased expression of immediate early response gene 3 protein promotes aggressive progression and predicts poor prognosis in human bladder cancer. BMC Urol. 2018;18(1):82.

32. Arlt $\mathrm{A}$, Schafer $\mathrm{H}$. Role of the immediate early response 3 (IER3) gene in cellular stress response, inflammation and tumorigenesis. Eur J Cell Biol. 2011;90(6-7):545-52

33. Mattila MM, Ruohola JK, Karpanen T, Jackson DG, Alitalo K, Harkonen PL. VEGF-C induced lymphangiogenesis is associated with lymph node metastasis in orthotopic MCF-7 tumors. Int J Cancer. 2002;98(6):946-51.

34. Jones D, Pereira ER, Padera TP. Growth and immune evasion of lymph node metastasis. Front Oncol. 2018;8:36.

35. Lien MY, Tsai HC, Chang AC, Tsai MH, Hua CH, Wang SW, Tang CH. Chemokine CCL4 induces vascular endothelial growth factor C expression and lymphangiogenesis by miR-195-3p in oral squamous cell carcinoma. Front Immunol. 2018;9:412.

36. Chen JC, Chang YW, Hong CC, Yu YH, Su JL. The role of the VEGFCNEGFRs axis in tumor progression and therapy. Int J Mol Sci. 2012;14(1):88-107.

\section{Publisher's Note}

Springer Nature remains neutral with regard to jurisdictional claims in published maps and institutional affiliations. 